\title{
RELAZIONARSI CON LA TERRA DI ORIGINE: SHIRIN RAMZANALI FAZEL, IGIABA SCEGO E MARYAM MAIO
}

\author{
ESTABLISHING RELATIONSHIPS WITH THEIR HOMELAND: SHIRIN
}

RAMZANALI FAZEL, IGIABA SCEGO E MARYAM MAIO

Caterina Duraccio

Universidad de Sevilla

\section{Riassunto:}

I rapporti politici tra Italia e Somalia hanno sempre destato particolare attenzione, in virtù del passato coloniale in comune. I movimenti diasporici tra i due Paesi, divisi in diversi momenti, sono stati i protagonisti del Novecento. In particolar modo, la diaspora somala assume un ruolo centrale all'interno della letteratura italiana dell'ultimo secolo, grazie alle produzioni autobiografiche di autrici come Shirin Ramzanali Fazel, Igiaba Scego e Maryam Maio. Le voci diasporiche entrano in relazione fra loro, offrendo una visione più completa di questo delicato periodo storico.

\section{Palabras clave:}

Diaspora Somala, Shirin Ramzanali Fazel, Igiaba Scego, Maryam Maio

\section{Abstract:}

Political relations between Italy and Somalia have always aroused attention because of their common colonial past. The diasporic movements between the two countries, divided at different times, have been the protagonists of the twentieth century. In particular, the Somali diaspora assumes a central role within the Italian literature of the last century, thanks to the autobiographical productions of authors such as Shirin Ramzanali Fazel, Igiaba Scego and Maryam Maio. The diasporic voices establish a relationship between them, offering a more complete vision of this sensitive historical period.

\section{KEYWORDS:}

Diaspora Somala, Shirin Ramzanali Fazel, Igiaba Scego, Maryam Maio 


\section{LA DIASPORA SOMALA IN ITALIA}

La diaspora somala occupa un vasto spazio nell'evoluzione della storia italiana contemporanea. L'esodo massivo dal paese africano attraversa diverse fasi, che dipendono dagli eventi storici che lo caratterizzano nei diversi anni, e pertanto non è una questione che si possa affrontare in modo unitario ed omogeneo. È dunque necessario definire i confini temporali delle rispettive fasi e tracciarne le rotte e i motivi. A grandi linee, è possibile distinguere tre momenti principali della diaspora somala in Italia: 1) gli anni '50, quando la Somalia era sotto il governo dell'AFIS (Amministrazione Fiduciaria dell'Italia in Somalia); 2) gli anni '70, con la dittatura di Siad Barre; 3) dagli anni '90 in poi, in seguito allo scoppio della guerra civile. I primi due periodi sono caratterizzati da un movimento migratorio quasi esclusivamente diretto verso l'Italia, il terzo assume tratti più estesi e i flussi in uscita si rivolgono a tutta l'Europa (Svezia e Inghilterra fra le prime). Il dominio coloniale italiano, che ha occupato i territori dell' Africa Orientale dall' 800 alla prima metà del ‘ 900 , ha imposto la lingua italiana come idioma principale, costruendo scuole ed enti che promuovessero il sistema linguistico e culturale della Madre Patria. La conoscenza della lingua italiana è stata il motivo principale della scelta del Bel Paese per le prime due fasi della diaspora. Ciò nondimeno, l'analisi dei diversi periodi non può prescindere dalle differenze che intercorrono tra gli stessi soggetti in movimento. Il viaggio, la migrazione e la diaspora sono elementi che si intrecciano in maniera consapevole all'interno della comunità. La seconda fase, però, è caratterizzata dall'esodo involontario di tutti i figli italo-somali, nati da coppie miste e dimenticati dalla Storia e dalla storiografia. Se in linea generale il periodo coloniale in Africa è poco conosciuto e studiato, quello dell'AFIS (Amministrazione Fiduciaria dell'Italia in Somalia), è un tentativo del tutto sconosciuto e oscurato. Negli anni '50 l'Organizzazione delle Nazioni Unite concesse all'Italia il governo politico e il controllo economico sulla Somalia. In via teorica, il compito dell'AFIS è quello di trasformare il paese dell' Africa Orientale in uno stato democratico e indipendente. La scelta di affidare l'amministrazione agli ex-coloni non è molto comune nei processi di decolonizzazione e ha dato vita ad una nuova fase del colonialismo italiano in Somalia. Molti degli italiani stanziati sulle coste somale continuarono ad abitarci, alimentando tensioni e differenze nella trama del tessuto sociale africano ${ }^{1}$. Prima della decisione dell'Onu, l'11 maggio 1948, alcuni gruppi armati somali entrano nel quartiere italiano e massacrano i residenti. Il clima sociale di quegli anni, dunque, è caratterizzato da una costante tensione tra le parti che ha generato insicurezza e paura in tutti gli abitanti di Mogadiscio. La nuova amministrazione è molto simile a quella precedente fascista e imperiale, di cui mantiene ideologia e pratiche. Uno dei temi cardine della relazione italo-somala, dagli anni del fascismo a quelli dell'AFIS, è la continuità dei rapporti di madamato tra i militari italiani e le, spesso giovanissime, ragazze somale. Durante 
il periodo coloniale fascista, il madamato (o madamismo) è stata una pratica molto comune, nelle colonie eritree prima e in tutta l'Africa Orientale Italiana poi. Questa questione, estremamente controversa, è rientrata nel dibattito contemporaneo in seguito alla proposta dei movimenti femministi italiani di riposizionare storicamente e culturalmente la figura di Indro Montanelli. Il giornalista, impiegato nelle campagne coloniali in Africa negli anni '40, aveva 27 anni quando sposò una giovanissima donna somala, la dodicenne Destà. La ragazza aveva tutti i doveri di una giovane moglie ma era completamente sprovvista dei diritti. Accusato diverse volte di pedofilia, Indro Montanelli pubblica sulla sua rubrica La Stanza, nel Corriere della Sera, il 12 febbraio 2000, un articolo in cui prova a spiegare la sua relazione con Destà:

Completamente frastornato dal nuovo ambiente (arrivavo da Parigi), mi presentai al comandante di Battaglione, Mario Gonella, un piemontese di lunga e brillante esperienza coloniale, che mi diede alcuni ordini, ma anche alcuni consigli sul modo di comportarmi con gl'indigeni e con le indigene. Per queste ultime, mi disse di consultarmi col mio sciumbasci, il più elevato in grado della truppa [...]. Si trattava di trovare una compagna intatta per ragioni sanitarie (in quei Paesi tropicali la sifilide era, e credo che ancora sia, largamente diffusa) e di stabilirne col padre il prezzo. [...] La ragazza si chiamava Destà e aveva 14 anni: particolare che in tempi recenti mi tirò addosso i furori di alcuni imbecilli ignari che nei Paesi tropicali a quattordici anni una donna è già donna, e passati i venti è una vecchia. Faticai molto a superare il suo odore, dovuto al sego di capra di cui erano intrisi i suoi capelli, e ancor di più a stabilire con lei un rapporto sessuale perché era fin dalla nascita infibulata $[\ldots]$.

Le affermazioni del giornalista da un lato rispecchiano appieno lo spirito coloniale e l'idea di una doppia superiorità (di razza e di genere) e dall'altro offrono una fotografia del fenomeno del madamato. Un altro aspetto importante è il perpetrare l'ipersessualizzazione della donna nera che "a quattordici anni una donna è già donna, e passati i venti è una vecchia" ${ }^{2}$. Questa concezione della presunta differenza biologica è quindi funzionale alla giustificazione e alla legittimazione di puri atti che in Madre Patria sarebbero stati condannati come pedofilia. La convivenza tra l'uomo italiano bianco e la donna somala nera non ha mai rappresentato un'unione ugualitaria ma è sempre stata a sfavore della seconda, il cui unico compito era la piena e totale soddisfazione dei bisogni maritali. Il madamato, pratica portata avanti per tutti i decenni della colonizzazione e rivendicata dagli ufficiali coloni, non era ben vista dal governo centrale poiché, nella maggior parte dei casi, queste relazioni portavano alla nascita di figli meticci. Come si può immaginare, il regime fascista non era ben disposto ad accettare la commistione della razza bianca con quella indigena e ha cercato di mettere un freno all'espansione del meticciato. A questo punto è però necessaria una precisazione: l'interesse, da parte dell'autorità italiana, non era chiaramente rivolto all'interruzione dello sfruttamento

2 Montanelli non fu mai chiaro sull'età precisa di Destà. Tuttavia, nelle ultime interviste dichiarò che la ragazza avesse dodici anni al tempo della loro conoscenza. 
sessuale delle donne somale nei rapporti di madamato, che non furono mai realmente scoraggiati. L'intenzione reale era esclusivamente quella di preservare la razza bianca e l'idea di un popolo puro. Infatti, mentre l'immaginario collettivo della donna africana selvaggia e sessualmente disinibita, rimaneva in voga per tutti gli anni -e anche oltre- del colonialismo, si inasprivano le sanzioni ai danni del meticciato. Secondo una legge del 30 dicembre 1937-XVI, n.2590 denominato "Sanzioni per i rapporti di indole coniugale fra cittadini e sudditi", "il cittadino italiano che nel territorio del Regno e delle Colonie tiene una relazione di indole coniugale con una persona suddita dell' Africa Orientale Italiana o assimilata è punito con la reclusione da uno a cinque anni" ${ }^{3}$. Queste leggi sono rimaste valide per tutto il periodo coloniale, a riconferma che l'Amministrazione Fiduciaria era in continuità con le pratiche imperiali fasciste. Nonostante le rigide normative, le coppie clandestine italo-somale aumentavano e, di conseguenza, si registrava un aumento di figli illegittimi, spesso non riconosciuti dai padri italiani. Abbandonati alle madri ancora adolescenti, i bambini venivano generalmente affidati a strutture cattoliche che si occupavano della loro educazione fino al compimento della maggiore età. Negli anni '50 si assiste ad un aumento delle infrastrutture cattoliche nelle terre d'oltremare, finanziate con fondi pubblici stanziati per la costruzione di scuole, rifugi e case-famiglia dove accogliere ed educare i nuovi italiani. Con il raggiungimento dell'indipendenza il $1^{\circ}$ luglio 1960 termina il controllo dell' AFIS ma molti italiani decidono di rimanere a Mogadiscio e, più in generale, sulle coste somali. Nonostante la fine formale e definitiva del periodo coloniale, l'attitudine degli italiani nei confronti degli abitanti locali continua a riprodurre rapporti di dominio e sottomissione. La donna africana continua ad incarnare il mito esotico e selvaggio di un corpo da addomesticare e da educare. Sparite le istituzioni italiane cattoliche, i figli delle coppie miste hanno solo due possibilità: la prima è quella di essere riconosciuti da entrambi i genitori, formare parte di un nucleo familiare legale e riconosciuto, godendo di tutti i diritti che ne derivano; la seconda -e quella più comune- è quella di non essere riconosciuti dai padri italiani e, pertanto, essere senza nome, i figli meticci della società coloniale. Affidarli all'educazione cattolica, pertanto, è l'unico modo che queste adolescenti hanno per poter garantire un'istruzione e un futuro migliore. Dopo l'indipendenza, intorno ai primi anni '60, le strutture cattoliche, con tutti gli affidatari e gli affidati, hanno l'ordine di lasciare l'Africa e rimpatriare. Una volta tornati in Italia, però, questi bambini non vengono riconosciuti come italiani ma categorizzati come razza afro-europea e ricollocati in apposite strutture. Allo stesso tempo, i figli riconosciuti (e quindi legittimi) rimasti in Somalia, subiscono la dittatura di Siad Barre che non li riconosce e, oltre a non godere dei diritti dei somali, non possono inserirsi nel mondo lavorativo. Emarginati ed isolati da entrambi i paesi, gli italo-somali nati da relazioni di madamato o clandestine, sono i grandi dimenticati

3 Cfr. Banti, Alberto Mario (2010) Le questioni dell'età contemporanea. Roma-Bari: Laterza. 
dalla storia italiana. Questa comunità, seppur minore, ha determinato e fa tuttora parte della nostra struttura sociale, eppure non godono di alcun riconoscimento. La storia delle relazioni italo-somale e dei rapporti di dominio e colonizzazione non sono mai stati approfonditi, né tantomeno conosciuti. A differenza delle altre grandi potenze europee che, alla fine della Seconda Guerra Mondiale, hanno riconosciuto gli errori dei propri processi coloniali, l'Italia non ha mai ammesso le proprie responsabilità politiche, né tantomeno le conseguenze prodotte dalla gestione dell' AFIS.

\section{LE RELAZIONi ITALO-SOMALE NEI ROMANZI DELLA DIASPORA}

L'enorme assenza storiografica dei rapporti tra Italia e Somalia è al centro del dibattito, apertosi nell'ultimo decennio, sugli studi e la letteratura postcoloniale e della diaspora italiana. Per il pieno utilizzo del termine "postcoloniale", inteso come l'approccio critico alle grandi colonizzazioni, è stato dunque necessario approfondire e capire gli eventi del periodo coloniale italiano in Africa. Il primo apporto della letteratura postcoloniale italiana e di quella della diaspora è stato quello di rendere visibile e far conoscere ad un pubblico italiano parte della sua storia. Numerose sono le voci che hanno animato il dibattito letterario e che hanno ricostruito, attraverso la memoria e la tradizione orale, la storia italo-somala ${ }^{4}$. Una delle scritture più forti e autorevoli nel campo degli studi e della letteratura postcoloniale è Igiaba Scego, che nei suoi romanzi introduce e propone una revisione critica della storiografia dominante. Con lei, le storie personali diventano uno strumento per raccontare la Storia, attraverso la narrazione biografica ed autobiografica vengono interpellati i personaggi storici reali, messi davanti alle proprie responsabilità. Inoltre, rende manifesta una parte della nostra storia che è stata oscurata per decenni. Lo sguardo critico della scrittrice è rivolto proprio a questo vuoto storico nella coscienza degli italiani:

Però su una cosa avevano ragione da vendere: 1 'Italia si era dimenticata del suo passato coloniale. Aveva dimenticato di aver fatto subire l'inferno a somali, eritrei, libici ed etiopi. Aveva cancellato quella storia con un facile colpo di spugna. Questo non significa che gli italiani siano stati peggio di altri popoli colonizzatori. Ma erano come gli altri. Gli italiani hanno stuprato, ucciso, sbeffeggiato, inquinato, depredato, umiliato i popoli con cui sono venuti in contatto. Hanno fatto come gli inglesi, i francesi, i belgi, i tedeschi, gli americani, gli spagnoli, i portoghesi. Ma in molti di questi paesi dopo la fine della Seconda guerra mondiale c'è stata una discussione, ci si è accapigliati, gli scambi di vedute sono stati aspri e impetuosi; ci si è interrogati sull'imperialismo e i suoi crimini; sono stati pubblicati studi; il dibattito ha influenzato la produzione letteraria, saggistica, filmica, musicale. In Italia invece silenzio. Come se nulla fosse stato. (Scego, 2010, p. 20)

4 PANDOLFO, Michele. 2017. «Gli italo-somali dell'Amministrazione Fiduciaria Italiana della Somalia (AFIS) », Diacronie [Online], N 30, 2 | 2017, documento 2, URL : http://journals.openedition.org/ diacronie/5651; DOI 10.4000/diacronie.5651 
Il silenzio delle istituzioni italiane rispetto alle responsabilità coloniali ha creato un enorme vuoto che stanno colmando le voci postcoloniali. Per mettere fine alla progressiva scomparsa della memoria coloniale italiana, nella seconda metà del ' 900 , nascono diverse associazioni: nel 1996 nasce l'ANCIS, acronimo di Associazioni Nazionale Comunità Italo-Somala e successivamente l'A.I.S.C.I.A (Associazione Italo-Somala Comunità Internazionale e Africana) che principalmente è un ente di volontariato. Tra gli altri, la prima ha come obiettivo "1) il sostegno al processo di pacificazione, sviluppo della Somalia e 2) la conoscenza dell'identità sociale e storica della Comunità Italo-Somala che si configura quale minoranza culturale e a tal fine dovrà assumere rilevanza lo studio sistematico della storia coloniale somala; il suddetto studio costituirà la base della relazioni bilaterali" ${ }^{5}$. L'ANCIS si propone come organismo mediatore nei rapporti tra l'Italia e la Somalia e ha come scopo principale la creazione di un ponte tra le due culture, attraverso la conoscenza della storia comune. A.I.S.C.I.A. è stata fondata da due donne, una italiana e una somala, e mira al sostegno psicofisico delle persone che hanno vissuto e affrontato la diaspora. Entrambe le associazioni, insieme alle comunità somale di varie regioni italiane, hanno costituito e costituiscono tutt'oggi il principale patrimonio di memoria storica della diaspora somala nel nostro Paese. Insieme agli enti associativi, gli studi postcoloniali e della diaspora hanno giocato un ruolo fondamentale nella diffusione storiografica di questi eventi. In particolare, il crescente sviluppo della letteratura italiana postcoloniale e quella della diaspora ha dato origine ad una considerevole varietà di contenuti. I temi che vengono primariamente affrontati interessano la costruzione dell'identità migrante, la riscrittura della Storia attraverso le narrazioni minori e la ricollocazione geoculturali dei soggetti diasporici. I testi qui presi in esame sono rappresentativi di ciascuna delle tre fasi della diaspora somala sopra descritti: Nuvole sull'equatore. Gli italiani dimenticati. Una Storia. di Shirin Ramzanali Fazel; La mia casa è dove sono di Igiaba Scego e Quando il sole splendeva sulla Somalia di Maryam Maio e Rita Corradi.

\subsection{NuVOLE SULL'EQUATORE}

Shirin Ramzanali Fazel è una scrittrice di origini somale arrivata in Italia negli anni '70. Suo marito è uno dei figli meticci dell'Africa coloniale con documenti italiani e, pertanto, costretti ad abbandonare la Somalia 6 . La scelta dell'Italia come meta, dunque, è stata la conseguenza naturale sia dal punto di vista burocratico che per affinità e conoscenze linguistiche. Il suo primo romanzo, Lontano da Mogadiscio (1994) è uno

5 Lo statuto dell'associazione è stato consultato al seguente indirizzo internet, URL: http://www.italosomali. org

6 Nel 1969 Siad Barre impose a tutte le persone non in possesso del passaporto somalo di lasciare il Paese. L'applicazione di questa normativa ha in gran parte determinato una forte ondata migratoria verso l'Italia, soprattutto dai figli delle coppie miste, con documenti italiani ma nella pratica, apolidi. 
degli scritti più importanti della letteratura italiana della migrazione ed è considerato, in un certo senso, l'apripista alla letteratura postcoloniale della diaspora somala.

Nuvole sull'equatore. Gli italiani dimenticati. Una storia, pubblicato nel 2010, è il suo secondo romanzo. Le intenzioni narrative sono chiare già dal titolo: tratta della storia di Giulia, figlia meticcia di una coppia italo-somala, dimenticata dalla Storia, invisibile nella storiografia. La storia viene raccontata in terza persona e ripercorre l'infanzia e l'adolescenza di Giulia a Mogadiscio, descrivendo e collocandola nel contesto politicosociale che la accompagna. La Storia, che fa da sfondo alla vicenda, irrompe nella vita dei protagonisti, cambiandone il naturale corso. Nel primo capitolo, uno dei pochi in cui è presente il padre Guido, gli eventi e le leggi somale determinano le abitudini dei cittadini:

\begin{abstract}
Guido dopo cena conduceva spesso la figlia al cinema. Era il suo passatempo preferito. [...] A Mogadiscio c'erano diverse sale cinematografiche. Padre e figlia, tenendosi per mano, raggiungevano il supercinema più vicino alla loro abitazione. Lungo la strada, sui marciapiedi, incontravano molte persone. Si conoscevano un po' tutti di vista. Un cenno di saluto con la mano o due battute facevano parte dell'abitudine locale. Le donne bianche portavano i tacchi a spillo e si reggevano al braccio dei loro uomini. Le coppie miste evitavano di tenersi a braccetto. Retaggio delle leggi fasciste che proibivano la mescolanza delle razze. E poi non era nell'usanza delle donne somale esporsi così sfacciatamente in pubblico. (Ramzanali Fazel, 2017, p. 8)
\end{abstract}

Da queste poche righe Shirin Ramzanali Fazel lascia intravedere le prime tensioni sociali che viveva Mogadiscio e l'emarginazione che subivano le coppie formate da un uomo italiano e una donna somala e, di conseguenza, la loro prole. Come osservato in precedenza, il meticciato era fortemente scoraggiato sia dal governo italiano che, in un secondo momento, da quello somalo, lasciando migliaia di persone in una condizione limbica. Il mestizaje culturale, etnico e linguistico che ha luogo in questi anni è indissolubilmente legato al femminile e ai rapporti di potere fra i generi. I ricordi della legislazione e della normativa fascista agiscono sulla vita degli abitanti di Mogadiscio ad anni di distanza dall'abbandono formale delle coste africane da parte degli italiani.

La complessa situazione politica somala ha generato dei movimenti indipendentisti che sognavano e pretendevano la liberazione della propria terra, sia dai retaggi dei domini coloniali (italiano e inglese) che dai dittatori locali. Amina, la madre di Giulia, attivista per l'indipendenza della Somalia è presentata come una donna forte e indipendente e con una grande sete di libertà. Quando il $1^{\circ}$ luglio 1960 la Somalia raggiunse la tanto agognata indipendenza, il marito Guido decide di trasferirsi a Nairobi, in Kenya. Il trasferimento è lo spartiacque nella loro vita, giacché Amina non accetta l'idea di aver lasciato la propria terra: 
Amina col passare dei mesi si sentiva sempre più insofferente. La convivenza con Guido diventava difficile e le frequenti discussioni portavano strascichi di periodi sempre più lunghi di silenzi tra loro. Dentro di sé la sensazione di vuoto e di solitudine cresceva. Guido aveva il suo lavoro, però le sere che egli rimaneva fuori per "affari" aumentavano. Ormai era conscia che si stavano allontanando anche fisicamente. Amina chiusa in quella casa si sentiva soffocare. Le mancava la sua Xamar, le amicizie di un tempo, la sua gente, il familiare vociare per le strade, la libertà di uscire a qualunque ora del giorno. (Ramzanali Fazel, 2017, p. 41)

Amina decide di mettere la sua libertà prima di ogni altra cosa e di tornarsene da sola a Mogadiscio. Nel frattempo, Giulia è rimasta a Nairobi con un padre sempre più assente e sente una forte mancanza degli affetti familiari, così decide di tornare in Somalia dalla madre. A partire da questo momento Guido sparisce completamente dalla narrazione e dalla vita della figlia, per poi ritornare nell'ultimo capitolo quando Giulia decide di andare a cercarlo. Appena arrivata però, si scontra con la realtà e affronta la delusione che prova nel vedere l'Italia da vicino:

Qui è tutto diverso da quello che si vedeva al cinema, la gente corre e non ha mai tempo. Molti purtroppo non sanno neppure dov'è la Somalia. Ci sono mendicanti bianchi che chiedono l'elemosina per le strade. I camerieri che servono ai tavoli, i facchini che trasportano le valigie, gli autisti, gli spazzini sono tutti bianchi. Non potevo crederci appena ho visto tutto questo. Non è vero che sono tutti ricchi in Italia, come volevano farci credere. (Ramzanali Fazel, 2017, p. 200)

Mentre in Africa Orientale la propaganda dell'Italia imperiale raccontava una Madre Patria libera dalla povertà, democratica ed ugualitaria, Giulia ne ritrova tutt'altra immagine. Davanti agli occhi della protagonista si sgretola la memoria dei racconti e si ricostruisce la realtà effettiva della società italiana. Il viaggio di Giulia nella patria del padre assume il valore della ricerca identitaria: la conoscenza della cultura paterna cela la pretesa di ricostruire una parte di sé. L'asimmetria tra i racconti di un'Italia ricca e benevola e la realtà con cui si è scontrata la giovane viaggiatrice determinano uno stordimento e uno straniamento dell'io straniero.

\subsection{LA MIA CASA È DOVE SONO}

Igiaba Scego, nata in Italia da genitori somali, è una delle voci più autorevoli della letteratura postcoloniale italiana, insieme a Shirin Ramzanali Fazel. Il suo romanzo La mia casa è dove sono (2010) è un viaggio introspettivo per i luoghi romani della diaspora somala. Ogni capitolo del libro, eccetto il primo e l'ultimo, porta il nome di un quartiere, un monumento o un punto di riferimento per la comunità italo-somala a Roma. Il romanzo si apre però nella casa di suo fratello a Manchester, con una riunione di famiglia. I membri, tutti di origini somale, hanno diverse nazionalità: italiana, inglese 
e finlandese. L'intera famiglia, infatti, era scappata dalla Somalia intorno agli anni '70, per opposizione politica al regime dittatoriale di Siad Barre. Le notizie intorno alla storia familiare vengono dilazionate nel corso del romanzo e servono a ripercorrere la storia della Somalia, gli esodi e la guerra, le conseguenze che hanno provocato nella vita quotidiana dei cittadini somali. Il racconto avviene in prima persona: la protagonista Igiaba, alter-ego letterario dell'autrice, propone di rimappare le città di Mogadiscio e di Roma, per riscrivere una storia che sia comune e condivisa. Nella cucina inglese la protagonista è accompagnata dal fratello, dal cugino e dal nipotino. Insieme ricordano la vecchia Mogadiscio, quella dell'infanzia per alcuni di loro e quella delle storie familiari per altri. Nel tentativo di disegnare la mappa della città si accorgono dei vuoti e dell'impossibilità di tracciarne i profili poiché la loro Xamar è probabilmente cambiata:

Una città morta... Tante città muoiono. Tali e quali a noi. Muoiono come qualsiasi organismo. Muoiono come gli gnu, le zebre, i bradipi, le pecore e gli esseri umani. Ma nessuno fa mai un funerale a una città. Nessuno ha fatto il funerale di Cartagine. Nessuno quello di New Orleans. Nessuno quello di Kabul, di Baghdad o di Portau-Prince. E nessuno ha mai pensato di commemorare Mogadiscio. Lei è morta. E qualcosa di diverso è sorto sopra le macerie. Non abbiamo nemmeno avuto il tempo di elaborare il lutto. Quando muore una città non ti danno nemmeno il tempo di pensare. Ma quel dolore è un cadavere, si decompone dentro di te e ti infesta di fantasmi. (Scego, 2010, p. 27)

Mogadiscio non esiste più. È un luogo della memoria e dell'anima che non trova spazio nel reale. La città ha subito le conseguenze del susseguirsi di cambiamenti politici: è stata distrutta e ricostruita senza dare ai cittadini la possibilità di elaborarne le trasformazioni. Ancora di più per gli esuli somali che, non avendole vissute, restano in un limbo atemporale in cui c'è una profonda asimmetria tra il passato e il presente. La protagonista del racconto prova a riunire le reminiscenze geografiche di Mogadiscio con le strade conosciute di Roma, disegnando un nuovo luogo, risultato e custode della sua duplice identità culturale.

La mia casa è dove sono presenta in diversi momenti la critica alla storiografia italiana, complice dell'oblio delle vicende coloniali:

L'Italia stava dappertutto nei nomi delle vie, nei volti dei meticci rifiutati. E l'Italia non ne sapeva niente, non sapeva delle nostre vie con i suoi nomi, dei nostri meticci con il suo sangue. In Italia alcune vie hanno i nomi dell' Africa. A Roma addirittura c'è il quartiere africano. In viale Libia, ti dice qualche romano, ci sono bei negozi di abbigliamento, ci puoi fare qualche buon affare. Ma poi? Poi niente. Vanno in viale Libia a comprarsi un maglione. Vivono in via Migiurtinia o si baciano in viale Somalia. Però ignorano la storia coloniale. Non è colpa loro: a scuola mica le impari queste cose. Siamo stati bravi, ti dicono, abbiamo fatto i ponti o le fontane. Il resto lo si ignora, perché non lo si insegna. (Scego, 2010, p. 30) 
Il richiamo al meticciato dimostra la centralità della questione nella storia italosomala e la mancanza di una giusta assunzione di responsabilità politiche da parte del governo italiano. Dal punto di vista della narrazione storica, la relazione tra in due Paesi è a senso unico: mentre la Somalia conserva l'eredità coloniale italiana, rivive il dolore delle violenze e delle oppressioni, mantiene ancora oggi l'insegnamento dell'italiano in alcune scuole; l'Italia non mostra nessuna conoscenza e nessun interesse non solo nella storia somala ma anche in quella italiana. Sarebbe - e in passato lo è stato- un errore considerare il dominio italiano a Mogadiscio una storia esclusivamente africana, altrettanto grave sarebbe relegarla ed affidarla unicamente agli studi postcoloniali. Al contrario, bisognerebbe mirare all'introduzione dello studio di tutto il periodo coloniale italiano (dalle prime esplorazioni della seconda metà dell'Ottocento fino all'inizio della seconda metà del Novecento) come parte integrante della nostra storia. Questa operazione sarebbe funzionale a normalizzare la duplice identità di una grande comunità che, non solo è presente nel territorio italiano, è italiana a tutti gli effetti.

2.3 Quando Il SOle SPlendeva Sulla Somalia

Myariam Maio, nata a Mogadiscio, è una delle due fondatrici dell'A.I.S.C.I.A. e attivista della Comunità Italo-Somala. Arriva in Italia nel 1991, in seguito allo scoppio della guerra civile in Somalia. Protagonista diretta della terza fase della diaspora, Maryam Maio scrive Quando il sole splendeva sulla Somalia nel 2016: un romanzo dai tratti autobiografici che ripercorre i primi trent'anni della sua vita a Mogadiscio. Scritto a quattro mani con Rita Corradi, il testo approfitta della narrazione autobiografica per colmare quei vuoti della Storia, che spesso ignora la comunità minori. Raccontare la Somalia ad un pubblico italiano è uno dei principali motori del romanzo ed è funzionale al riscatto della memoria individuale e collettiva del suo popolo.

Quando il sole splendeva sulla Somalia è scritto in prima persona e si apre con l'arrivo di Maryam, insieme alla figlia di due anni, a Roma nel quartiere di Montesacro. La narrazione si concentra principalmente sui sentimenti della protagonista e su cosa significasse, sul piano personale, l'abbandono forzato della propria patria:

\begin{abstract}
Ero confusa, tutto si era svolto troppo velocemente per poter capire cosa avrei fatto della mia vita da quel momento in poi. Avevo conservato la pelle, e soprattutto avevo portato in salvo mia figlia, e al momento ciò mi bastava. Di una cosa, però, avevo la certezza: il mio cammino non era stato fermato con un punto. Non avrei proseguito sulla stessa riga, e nemmeno in quella a capo. Ero approdata a un capitolo del tutto nuovo e pieno di incognite. Tornare indietro sarebbe stato impossibile. (Maio \& Corradi, 2016)
\end{abstract}

Il 1991 rappresenta un punto di non ritorno: a partire da quel momento la patria e la casa hanno smesso di esistere nelle forme conosciute. La ricollocazione geografica 
forzata, l'abbandono della terra natia e la perdita degli affetti conducono alla dislocazione del soggetto migrante. Gli spazi che si vivono e si attraversano a partire dal momento dell'esodo, diventano luoghi ibridi dove convivono le diverse identità (Bhabha: 2006). Il trasferimento in un altro paese e la conseguente assimilazione della sua cultura costruisce e fa parte dell'identità migrante, sede di entrambe le culture di appartenenza. Maryam Maio non lascia che la storia sia un mero sottofondo descrittivo ma la rende viva e attiva, partecipe e responsabile degli eventi:

\begin{abstract}
Tra la metà e la fine dei Settanta, e in special modo nel biennio' $766^{\prime} 77$, lo spauracchio della guerra control'Etiopia, che continuava a trascinarsi dopo il disastroso conflitto per il controllo della vasta regione dell'Ogaden, persa dopo il colonialismo - che fu una delle questioni più spinose e pericolose per il delicato equilibrio tra le due superpotenze in piena Guerra fredda- era una spada di Damocle che pendeva sulle teste di molti giovani in armi. Approfittando del fatto che l'Etiopia fosse in conflitto con l'Eritrea, diventata poi indipendente, Barre era tornato ad attaccare di nuovo; voleva a tutti i costi ridisegnare il confine originario pre-coloniale, riguadagnando territorio sull'Ogaden. I ragazzi quindi, già a disposizione dei militari, dovevano essere addestrati alla guerra. (Maio \& Corradi, 2016, p. 62-63)
\end{abstract}

Le informazioni fornite dall'autrice riguardano strettamente la comunità somala: da questi eventi scaturiva l'inizio del governo dittatoriale di Siad Barre. Una storia che il popolo italiano dovrebbe conoscere poiché è il risultato di decenni della sua dominazione e amministrazione. Per lo spirito e i presupposti che l'autrice introduce nel romanzo è più opportuno parlare di letteratura della migrazione della diaspora somala poiché è scevro della critica alla società coloniale e non rivendica l'appartenenza al soggetto postcoloniale. In ogni caso, Quando il sole splendeva sulla Somalia contribuisce alla ricostruzione delle storie delle relazioni italo-somale, raccontando eventi e vicende proprie del suo popolo.

\title{
3. Conclusioni
}

I romanzi presi in analisi nel seguente articolo sono accomunati da tre caratteristiche principali: la scrittura autobiografica, l'esperienza diasporica e, non ultima, la questione di genere. Questi elementi sono profondamente interconnessi tra loro poiché attraversano la totalità della biografia delle rispettive autrici. Il protagonismo del femminile nella letteratura autobiografica ha occupato gran parte del dibattito letterario e culturale del Novecento, imponendo nuove pratiche di scrittura, che non soltanto si pongono in opposizione al primato maschile, ma che fuoriescono dal sistema di definizioni binarie ed introdurre una molteplicità di voci e di esperienze (Mapelli, 2019).

Scrivere al femminile e del femminile è dunque uno strumento per sovvertire l'ordine androcentrico, suggerendo prospettive ed esperienze plurali. Nell'esperienza 
migratoria e diasporica l'autobiografia femminile assume un ruolo centrale ed articolato. Dal punto di vista storiografico, raccontare la diaspora significa aggiungere un tassello alla narrazione principale, colmando i vuoti lasciati dalla storiografia tradizionale. Dal punto di vista letterario, le tre autrici danno priorità all'esperienza femminile, utilizzando il proprio sentire come mezzo per descrivere le esperienze cruciali che le hanno attraversate. Ne La mia casa è dove sono la necessità di scrivere dell'autrice trova luogo nella rimappatura di Mogadiscio, attraverso i ricordi e la propria genealogia. Intorno alla mappa della città somala si ricostruisce la storia di generazioni di donne e delle loro migrazioni. La volontà di rivivere e riscrivere Mogadiscio è presente in tutti e tre i romanzi, testimonianza della necessità di rendere visibile la propria storia e quella della propria terra.

Il dibattito intorno alla diaspora somala e la letteratura da essa prodotta, è uno dei centri di interesse degli studi comparati e letterari degli ultimi decenni. Le voci diasporiche irrompono nella storiografia, ampliandola e arricchendola, aggiungendo non solo un nuovo punto di vista ma aiutando a ricostruire il mosaico di una Storia complessa e articolata. Le relazioni tra i due Paesi hanno determinato una serie di fenomeni, tra cui il madamato e il meticciato, che hanno inesorabilmente cambiato la struttura sociale italiana. Lo studio di tali rapporti è dunque fondamentale per poter conoscere e capire i cambiamenti storico-politici e sociali della nostra nazione. I tre romanzi presi in analisi descrivono le tre diverse fasi della diaspora somala, analizzando le influenze che questa situazione politica ha avuto nei protagonisti. Tutti e tre sono accomunati dal sentimento della perdita, l'abbandono della terra natale e degli affetti e soprattutto dalla presa di coscienza (da parte delle protagoniste) di un'identità multipla ed ibrida che si basa sulla convivenza tra le due culture.

\section{RIFERIMENTI BIBLIOGRAFICI}

Albertazzi, S., 2000. Lo sguardo dell'altro. Le letterature postcoloniali, Roma, Carocci, 2000.

Albertazzi, S., vecchi, R., (eds), Abbecedario postcoloniale: dieci voci per un lessico della postcolonialità, Macerata, Quodlibet, 2001.

Banti, A. M., Le questioni dell'età contemporanea. Roma-Bari, Laterza, 2010.

Benvenuti, G., ceserani, R., La letteratura nell'età globale, Bologna, Il Mulino, 2012.

Bhabha, H., The Location of Culture, London/New York, Routledge; trad.it. 2006. I luoghi della cultura. Roma, Carocci, 1994.

Braidotti, R., Soggetto nomade. Femminismo e crisi della modernità, Firenze, Donzelli, 1995

Braidotti, R., Trasposizioni. Sull'etica nomade. Roma, Luca Sossella editore, 2007. 
Chakrabarty, D., Provincializzare 1'Europa. Roma, Meltemi, 2004.

Chambers, I., curti, L. La questione postcoloniale: cieli comuni, orizzonti divisi. Napoli, Liguori, 1997.

Chambers, I., Paesaggi migratori: cultura e identità nell'epoca postcoloniale. Genova, Costa \& Nolan, 1996.

Comberiati, D. La quarta sponda. Scrittrici in viaggio dall'africa coloniale all'italia di oggi, Roma, Caravan edizioni, 2009.

Curti, L. La voce dell'altra. Scritture ibride tra femminismo e postcoloniale, Roma, Meltemi, 2006.

De robertis, R. (ed)., Fuori centro. Percorsi postcoloniali nella letteratura italiana. Roma, Aracne editrice, 2010.

Del boca, A., Gli italiani in Africa Orientale. Roma-Bari, Laterza, 1984

Fazel, S. R., Lontano da Mogadiscio. Roma, DATANEWS, 1999.

Fazel, S. R., Nuvole sull'equatore. Gli italiani dimenticati dalla storia. Italia, Shirin Ramzanali Fazel, 2017.

Foucault, M., Sorvegliare e punire. Torino, Einaudi, 2005.

Fracassa, U., Patria e lettere. Per una critica della letteratura postcoloniale e migrante in Italia. Roma, Giulio Perrone, 2012.

Ghermandi, G., Regina di Fiori e di Perle. Roma, Donzelli, 2007.

Gnisci, A., La letteratura italiana della migrazione. Roma, Lilith, 1998.

Maio, M., Quando il sole splendeva sulla Somalia. Chieti, Solfanelli, 2016

Mellino, M., La critica postcoloniale. Decolonizzazione, postcolonialismo e cosmopolitismo nei postcolonial studies. Roma, Meltemi, 2005.

Mellino, M., Post-orientalismo. Roma, Meltemi, 2008.

Mezzadra, S., La condizione postcoloniale. Storia e politica nel presente globale. Verona, Ombre Corte, 2008.

Pandolfo, M. « Gli italo-somali dell'amministrazione Fiduciaria Italiana della Somalia (AFIS) », Diacronie [Online], N 30, 2 | 2017, documento 2, URL : http://journals. openedition.org/diacronie/5651; DOI : 10.4000/diacronie.5651

Parati, G., Migration Italy: The Art of Talking Back in a Destination Culture. Toronto, University of Toronto Press, 2005.

Said, E., Culture and Imperialism. New York, Alfred A. Knopf 2004.; trad.it. Cultura e Imperialismo. Letteratura e consenso nel progetto coloniale dell'occidente. Roma, Gamberetti, 2006.

Scego, I., La mia casa è dove sono. Milano, Rizzoli, 2010. 
Spivak, G., C., A critique of Postcolonial Reason. Towards a History of the Vanishing Present. Cambridge (Mass)-London, Harvard University Press, 1999, trad.it. Critica della ragione postcoloniale. Verso una storia del presente in dissolvenza. Roma, Meltemi, 2004.

Thiong'o n. W., Decolonizing the Mind. The politics of Language in African Literature. London, Heinemann1986 ; trad.it. Spostare il centro del mondo, Roma, Meltemi, 2000.

Tomasello, G. L'Africa tra mito e realtà. Storia della letteratura coloniale italiana. Palermo, Sellerio, 2004. 